\title{
Modeling and Synthesis of Multiport Transmission Line for Multichannel Communication
}

\author{
Jun Chen, Student Member, IEEE, and Lei He, Member, IEEE
}

\begin{abstract}
To overcome the limitations of traditional interconnects, multichannel interconnects that transmit signals via highfrequency carriers have recently been proposed and realized for intrachip and interchip communication. To efficiently design such transmission-line-based interconnects, this paper derives a closedform model for signal-to-noise ratio (SNR) considering multiple ports and branches, and proposes efficient figures of merit (FOMs) to minimize signal distortion. Experiments show that the SNR model is accurate compared to SPICE simulation and the signal distortion FOMs are effective. Using the proposed models, this paper further automatically synthesizes coplanar waveguides (CPWs) for radio-frequency ( $R F$ ) interconnects with capacitive couplers. The authors minimize the total interconnect area under the constraints of SNR and signal distortion. Compared to the published manual designs, the synthesized solutions can reduce up to $80 \%$ area. Furthermore, the optimized solutions vary greatly with respect to number of ports, frequency bands, topologies, and terminations, and therefore automatic synthesis is effective and necessary.
\end{abstract}

Index Terms-Design automation, integrated circuit interconnections, interconnect modeling, RF interconnect, signal distortion, signal-to-noise ratio.

\section{INTRODUCTION}

$\mathbf{T}$ RANSMISSION lines have long been used in on-board and in-package communication. For on-chip communication, traditional interconnects transmit baseband signals and are $R C$ dominant. They have inherent signal distortion and large $R C$ delay [1], and the delay cannot scale as well as transistor speed [2]. Recently, transmission lines have been applied to onchip signal and clock distribution, and can be categorized into three types. The first type of interconnects directly transmits baseband signals. For clock distribution, transmission lines with dedicated ground planes have been used to achieve 5-GHz on-chip global clock frequency [3]. Tunable transmission lines have been used for global clock distribution in a microprocessor [4]. A synthesis algorithm for transmission-line-based clock tree has been developed [5]. For signal transmission, the transmission line has been exploited for cache communication to narrow the speed gap between processors and caches [6], and the pulsed waveform in a transmission line has been used to

Manuscript received February 27, 2005; revised July 10, 2005 and August 10, 2005. This work was supported in part by the National Science Foundation (NSF) CAREER Award CCR-0401682, an SRC Grant 1100, a UC MICRO Grant sponsored by Analog Devices, Fujitsu Laboratories of America, Intel, and LSI Logic, and a Faculty Partner Award by IBM. This paper was recommended by Associate Editor W. Schoenmaker.

The authors are with the Electrical Engineering Department, University of California, Los Angeles, CA 90095 USA (e-mail: jchen@ee.ucla.edu; lhe@ee.ucla.edu).

Digital Object Identifier 10.1109/TCAD.2005.858272 transmit signals [7]. The advantage of such interconnects is that their transceivers are simple, but they have limited wire bandwidth because the interconnect is wide and hard to be shared between different transceivers. Besides, they operate at the base band and have similar limitations as traditional interconnects.

The second type of interconnects modulates the baseband signal with high-frequency carriers such as radio-frequency (RF) signals before transmission, and the signal is recovered by analog receivers. Same as the first type of interconnects, they have also been applied to both clock distribution [8], [9] and signal transmission [1], [10]. The signals in such interconnects are transmitted in high-frequency bands that have much smaller distortion across the bandwidth compared to interconnects transmitting signal in base band, and can be propagated at a velocity close to the speed of light [1]. Another advantage of such interconnects is that they can be shared by multiple communication channels at different frequency bands, and each frequency band can be further shared by multiple code division communication channels. Therefore, the interconnect can potentially have a large bandwidth and also be reconfigurable. With the features of multiple access, such interconnects often have multiple ports and may have multiple branches.

The third type of interconnects is a very special kind of transmission line for both clock generation and distribution, which has been studied recently in [11]-[13]. Different from traditional clocking schemes, they utilize the oscillation between intrinsic inductance and capacitance of transmission lines to generate and distribute high-frequency and low-power clock signals for the entire board or chip. The work in [12] is based on traveling wave and produces multiphase square clock waveform, and [11] and [13] are based on standing wave and produce single-phase sinusoidal clock waveform on board and on chip, respectively. With multiple sinks, these interconnects also have multiple ports and branches.

In this paper, we study the second type of interconnects, which can be called as multichannel interconnects with highfrequency carriers. But our models can also be applied to the analysis of other multiport interconnects such as the clock distribution network in [11] and [13]. Designs and fabrications of interconnects transmitting signals via high-frequency carriers have already been presented in the literature, including a $2-\mathrm{GHz}$ H-tree-type RF global clock network on a $34 \mathrm{~cm} \times 17 \mathrm{~cm}$ PCB board [8], a 2-cm-long on-chip interconnect transmitting a $1 \mathrm{~Gb} / \mathrm{s}$ signal via a $7.5-\mathrm{GHz}$ carrier [1], a $1.1-\mathrm{Gb} / \mathrm{s}$ onchip multiport RF interconnect via a 10-GHz RF carrier [14], [15], a multilevel signaling scheme for an on-chip RF interconnect with $2-\mathrm{Gb} / \mathrm{s}$ data rate [16], a $3-\mathrm{Gb} / \mathrm{s} \mathrm{RF}$ interconnect in 
three-dimensional (3-D) integrated circuit [17], [18], and multichannel multicarrier on-chip RF interconnects based on frequency division multiple access (FDMA) technique and code division multiple access (CDMA) technique [10], [19].

However, all these works focus on circuit design. No efficient computer-aided design techniques were presented and the aforementioned circuits are most likely designed manually. Manual design takes a long time and may result in unnecessarily large area as we will see later in this paper. Furthermore, system-scale designs as in [6] and [9] are too complex to design manually. To bridge this gap, efficient models and automatic synthesis methods are needed, and accurate models must consider multiple channels, multiple ports, and branches. In addition, because of the analog nature of the carrier and transceivers, the model should focus on the frequency-dependent signal-tonoise ratio (SNR) and signal distortion. Numerical methods such as circuit simulation, e.g., [20]-[23], and model order reduction, e.g., [24], [25], can provide generic solutions to the voltage response in time or frequency domain, but they are too time consuming to be used in automatic synthesis. Analytic methods are efficient and can be used in synthesis. However, existing analytic models such as [5] and [26]-[28] focus on delay and noise in the time domain (and for only two ports), and there exists no analytical model for frequency-dependent SNR and signal distortion in a multiport transmission line. Generally, no accurate yet efficient design automation techniques have been developed for such transmission lines.

In this paper, we present efficient models for interconnects carrying high-frequency carriers considering multiple frequency channels, multiple ports, and branches. We first derive an accurate model with linear complexity for the frequencydomain voltage response in branched transmission lines and then develop closed-form formulas for the amplitude of the signal and the reflection noise at receivers. We thus obtain the frequency-dependent SNR for each receiver. The SNR model is accurate compared to SPICE simulations. We further propose figures of merit (FOMs) to minimize the signal distortion in both signal phase and amplitude. The proposed SNR model and distortion FOMs can be applied to any multiband multiport transmission line carrying high-frequency carriers. As an example, we apply our models to the synthesis of coplanar waveguide (CPW) for on-chip multichannel RF communication under the constraints of SNR and signal distortion. We minimize the area of the interconnects with either perfect or imperfect terminations. The synthesis results demonstrate up to $80 \%$ less chip area compared to the published manual designs [10]. We also successfully synthesized an interconnection with multiple branches. All the designs have been verified with time-domain transient simulation, which further validates our models. The synthesized designs vary with respect to the topologies, the number of ports, carriers, and terminations, and therefore show the effectiveness and necessity of the automatic synthesis process.

The rest of the paper is organized as follows. In Section II, we present the model for SNR and FOMs of signal distortion for multiport transmission lines. In Section III, we automatically synthesize the CPW structure for RF interconnection. We conclude the paper in Section IV.

\section{Models for MultiPort Transmission Lines}

In this section, we develop the models for a multiport transmission line. We assume that the signal is transmitted via a carrier signal at a fixed frequency and develop our models in the frequency domain. To avoid ambiguity, in this work we define a frequency channel as an FDMA channel with a fixed carrier frequency, and a communication channel as a signal path from a transmitter to a receiver. Each communication channel has only one transmitter and one receiver. Each frequency channel can only have one transmitter, but it may have multiple receivers, and therefore can include multiple communication channels. We first show an accurate model for port voltage response, then develop a closed-form model for SNR of a single uniform transmission line, and finally extend the model to branched transmission line structures with multiple different branches. In addition, we propose metrics for signal distortion.

\section{A. Background of Transmission Line}

According to the general transmission line theories [29], [30], a transmission line can be described as

$$
\begin{aligned}
& \frac{\partial V}{\partial x}=-(R+j \omega L) I \\
& \frac{\partial I}{\partial x}=-(G+j \omega C) V
\end{aligned}
$$

where $R, L, G$, and $C$ are the unit length resistance, inductance, conductance, and capacitance of the transmission line, respectively. $G$ is usually very small and can be ignored. The characteristic impedance of the transmission line is

$$
Z_{0}=\sqrt{\frac{R+j \omega L}{G+j \omega C}} .
$$

The general solution to (1) and (2) is

$$
\begin{aligned}
V & =A \exp (-\gamma x)+B \exp (\gamma x) \\
I & =\frac{A}{Z_{0}} \exp (-\gamma x)-\frac{B}{Z_{0}} \exp (\gamma x)
\end{aligned}
$$

where $A$ and $B$ are determined by boundary conditions. In (4) and (5), the component of $\exp (-\gamma x)$ is the forward wave propagating in the positive direction of the $x$-axis and the component $\exp (\gamma x)$ is the backward wave propagating in the negative direction of the $x$-axis. $\gamma$ is the propagation constant of the transmission line and is defined as

$$
\gamma=\alpha+j \beta=\sqrt{(R+j \omega L)(G+j \omega C)}
$$

where $\alpha$ is the attenuation per unit length and $\beta$ is the phase shift per unit length.

The propagated wave is reflected at the terminations of the line. Assuming the impedance of the termination is $Z_{t}$ and the amplitudes of forward and backward waves are $A$ and $B$, then the reflection ratio is

$$
\Gamma=\frac{B}{A}=\frac{Z_{0}-Z_{t}}{Z_{0}+Z_{t}}
$$




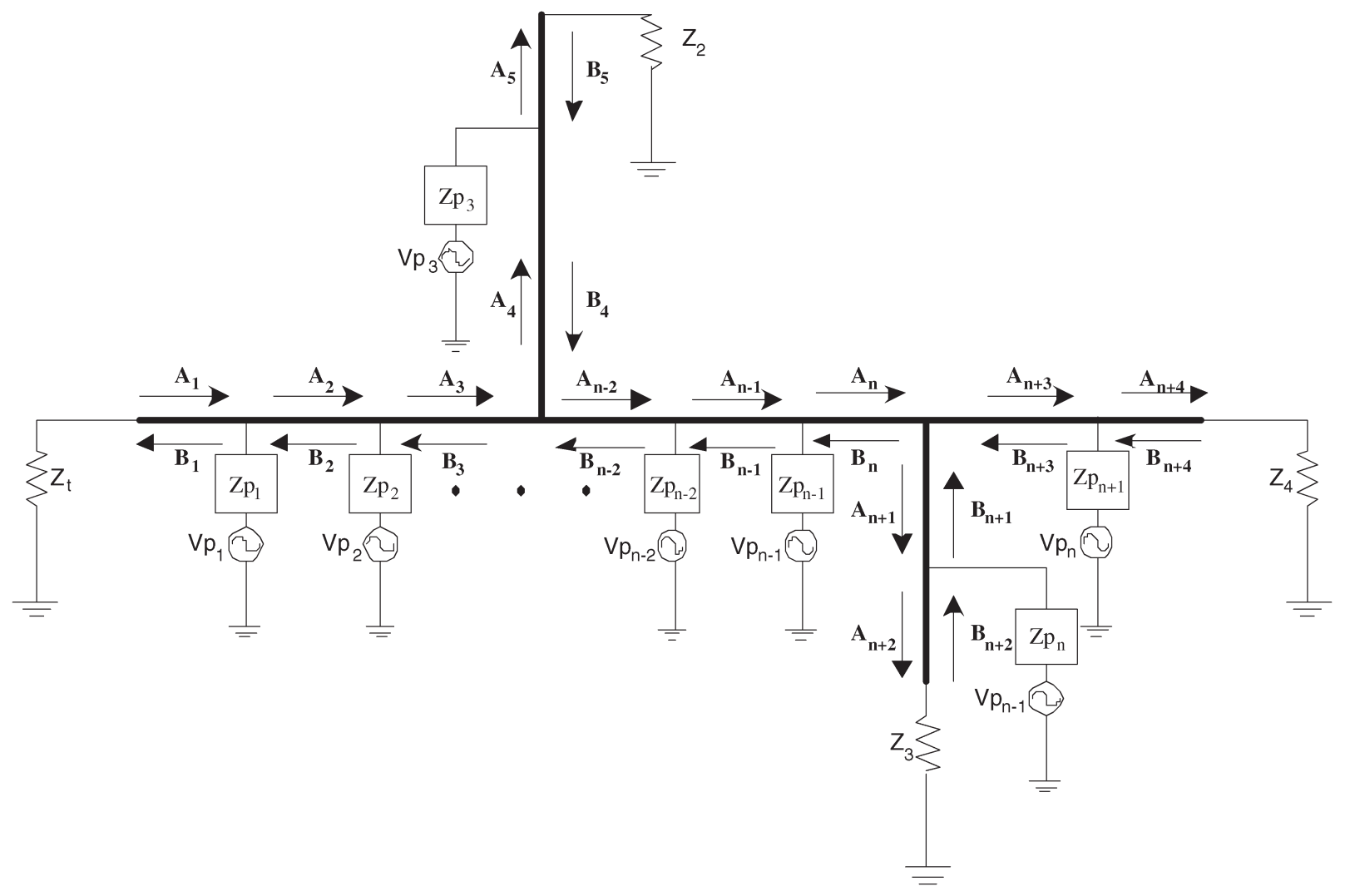

Fig. 1. Circuit model of multiport transmission line.

Reflections constitute part of the noise interfering with the propagated signal.

\section{B. Port Voltage Response}

Multiple ports connected to the transmission line introduce extra discontinuities and reflections. In Fig. 1, we show the interconnect model with multiple transceivers and branches. We assume linear transmitter and receiver models, and model each of them uniformly with an impedance and an ac voltage source, where the amplitude of the voltage source for a receiver is zero. The capacitive coupler is modeled as a lumped capacitor. Because the circuit is linear, according to superposition principle we can consider each frequency channel separately.

We consider three types of discontinuities of the transmission line structure: ports, branching points, and terminations. The segment between adjacent discontinuities is a continuous segment of transmission line, where the general solution of (4) and (5) still holds. The current and voltage between adjacent discontinuities $k$ and $k+1$ can be written as

$$
\begin{aligned}
& V_{k}(x)=A_{k} \exp \left(-\gamma\left(x-x_{k}\right)\right)+B_{k} \exp \left(\gamma\left(x-x_{k+1}\right)\right) \\
& I_{k}(x)=\frac{A_{k}}{Z_{0}} \exp \left(-\gamma\left(x-x_{k}\right)\right)-\frac{B_{k}}{Z_{0}} \exp \left(\gamma\left(x-x_{k+1}\right)\right)
\end{aligned}
$$

where $A_{k}$ and $B_{k}$ are the amplitudes of the forward and backward waves (see Fig. 1), $Z_{0}$ is the characteristic impedance of the transmission line, and $x_{k}$ is the location of discon-

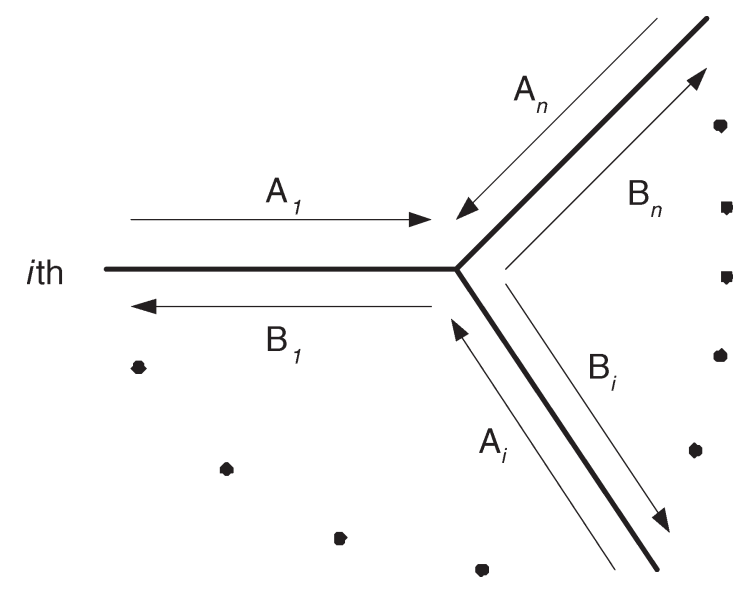

Fig. 2. Branching point.

tinuity $k . A_{k}$ and $B_{k}$ are to be determined by our voltage response model.

Each transmitter or receiver is a port to the interconnect. At a port $k$, by applying $\mathrm{KVL}$ and $\mathrm{KCL}$, we have

$$
\begin{aligned}
V_{k}\left(x_{k}\right) & =V_{k+1}\left(x_{k}\right) \\
Z p_{k}\left(I_{k}\left(x_{k}\right)-I_{k+1}\left(x_{k}\right)\right) & =V_{k}\left(x_{k}\right)-V p_{k}\left(x_{k}\right)
\end{aligned}
$$

where $Z p_{k}$ is the transceiver impedance and $V p_{k}$ is the transceiver voltage at port $k$.

At a branching point with $n$ branches connected as shown in Fig. 2, we have incoming waves $\left(A_{i}\right)$ and outgoing waves $\left(B_{i}\right)$ 


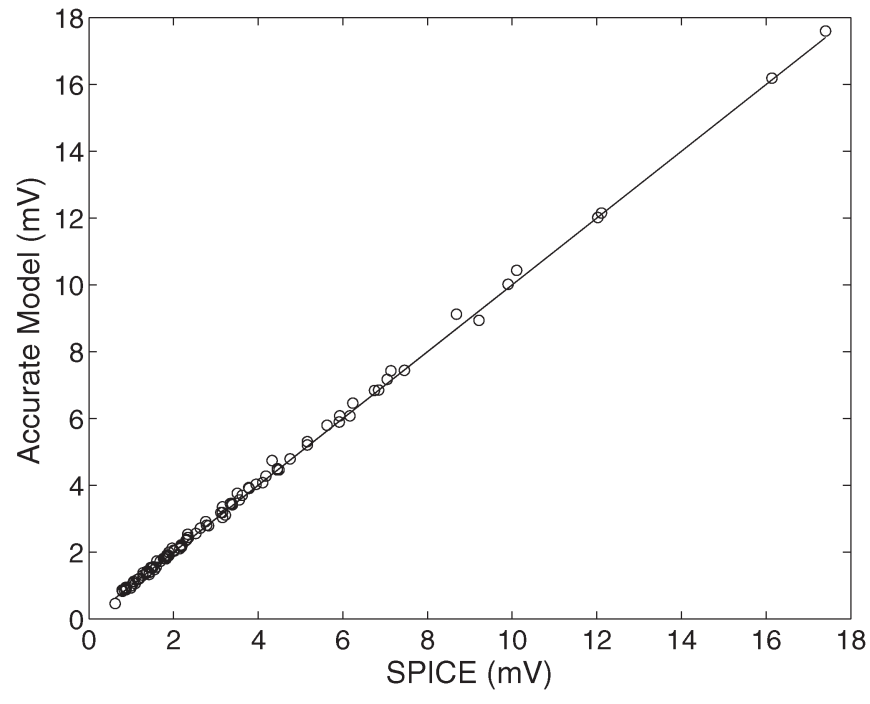

Fig. 3. Voltage comparison between multiport model and SPICE simulation.

on each connected branches, where $i=1,2, \ldots, n$. According to KCL

$$
\sum_{i=1}^{n}\left(\frac{A_{i}}{Z_{i}}-\frac{B_{i}}{Z_{i}}\right)=0
$$

where $Z_{i}$ is the characteristic impedance of branch $i$. Also, because the branches are connected at the branching point, for any pair of branches $i$ and $j$

$$
A_{i}+B_{i}=A_{j}+B_{j} .
$$

At the terminations of the transmission line, the voltage and current of the transmission line must satisfy

$$
V=Z_{t} I
$$

Assuming there are $n$ ports and $b$ branches, then there are $n+b$ segments of transmission lines and totally $2(n+b)$ unknown variables. Applying (10) and (11) to each port, (12) and (13) to each branching point, and together with (14) at each termination, we have $2 n+2 b$ linear equations. For example, for a two-port line, the system equations are shown as that in (15) at the bottom of the page. Because only neighboring segments have coupling terms, the matrix is a sparse band matrix, the equation set can be efficiently solved by Gaussian elimination method with a time complexity of $O(n)$. We compare our voltage response model with SPICE simulations in Fig. 3 for the voltage amplitude at different receivers. All SPICE simulations in this paper use a distributed $R L C$ model with one $R L C$ circuit for each wire segment of $5 \mu \mathrm{m}$. Other settings such as transceiver impedances and locations are randomly generated. The number of ports is between 10 and 100, and we randomly choose the communication channel for comparison. According to the figure, our model almost perfectly matches the SPICE simulations.

\section{Closed-Form SNR Model for Single Transmission Line}

To facilitate the computation of SNR and distortions, we develop models for signal and reflection noise amplitude at the receiver of one communication channel. We first consider a single uniform transmission line without any branches in this section. Obviously, each transmitter or receiver can only transmit signals on one frequency channel. Signals in other channels are filtered out by the receiver. Based on the superposition principle, we can compute the waveform of each frequency channel separately. We assume the transceiver impedances are much larger than $Z_{0}$ so that the reflections from the ports are small and the transmission line is not disturbed much by the shunt impedances. With this assumption, we first derive a simplified model considering only the transmitter and the receiver but ignoring other ports, and then extend the model to consider the effects of other ports and termination reflections. We also separate the computation of propagated signal from that of noise because the circuit is linear and the superposition principle can be applied.

1) Isolated Communication Channel: In this model, we consider one transmitter and one receiver but ignore the effect of other ports and terminations. We also only consider firstorder effects on the signal at the receiver, which means we only consider the reflected wave from only one reflection, because waves after multiple reflections will have very small amplitudes. Under these assumptions, following the same notation as in Section II-B and assuming the transmitter at port 1 and the receiver at port 2 , the simplified model for the transmitter port and the receiver port is shown in Fig. 4. According to (8)-(11), at the transmitter port, we obtain

$$
A_{2}=\frac{\frac{Z_{0}}{2}}{\frac{Z_{0}}{2}+Z_{s}} V s
$$

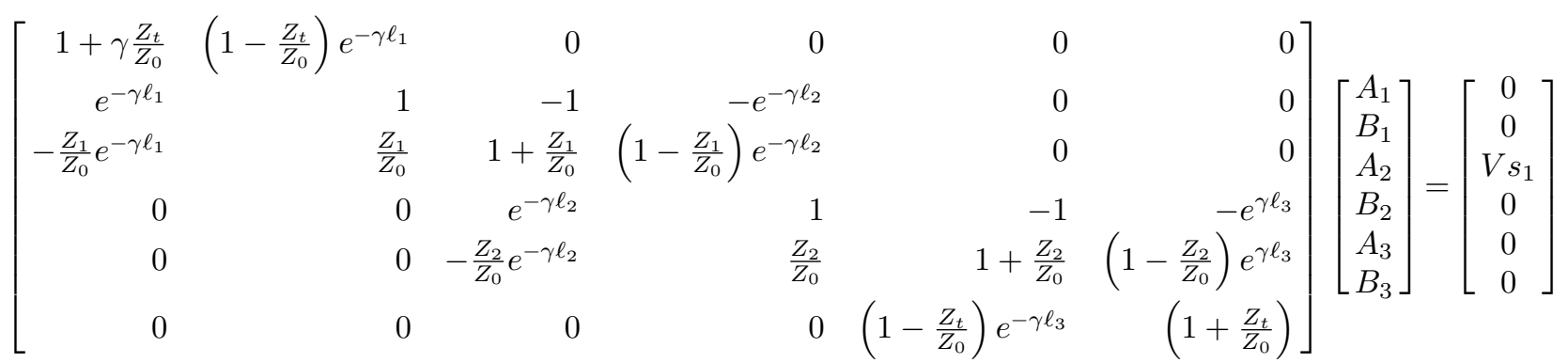




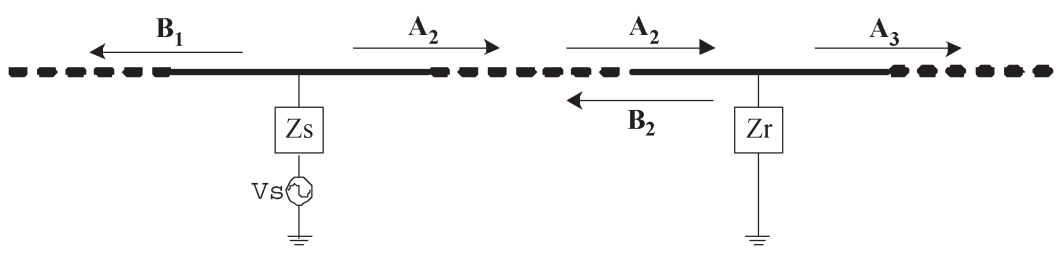

Fig. 4. Simple model for transmitter and receiver port.

where $Z_{s}$ and $V_{s}$ are the impedance and voltage at the transmitter port, respectively. $B_{2}$ is ignored at the transmitter port because we only considered the first-order effect on the signal at the receiver. $A_{2}$ is the signal amplitude coupled onto the transmission line that propagates toward the receiver. From (16), we can see that the larger $Z_{0}$ is, the larger the coupled signal is. Similarly, at the receiver, we have

$$
\begin{aligned}
A_{3} & =\frac{2 A_{2}}{\frac{Z_{0}}{Z_{r}}+2} \exp (-\gamma \ell) \\
B_{2} & =-\frac{A_{2}}{1+\frac{2 Z_{r}}{Z_{0}}} \exp (-\gamma \ell)
\end{aligned}
$$

where $Z_{r}$ is the shunt impedance at the receiver port and $\ell$ is the distance between the transmitter and the receiver. $A_{3}$ is the signal after the reflection at the receiver port. Since $A_{2}$ has been solved in (16), the voltage across the receiver input resistance $R_{r}$ is

$$
V_{r}=\frac{R_{r}}{Z_{r}} A_{3}=\frac{\frac{R_{r} Z_{0}}{2}}{\left(\frac{Z_{0}}{2}+Z_{r}\right)\left(\frac{Z_{0}}{2}+Z_{s}\right)} \exp (-\gamma \ell) V_{s}
$$

which is the signal voltage at the RF receiver.

2) Effect of Multiple Ports: In this section, we further consider the effect of other ports and extend the model in Section II-C1. When a propagating wave passes through a port, part of the signal is reflected according to (17) and (18). The situation is similar to that at a receiver in Section II-C1. According to (17), the transmission rate for port $k$ is

$$
\xi_{k}=\frac{2}{\frac{Z_{0}}{Z p_{k}}+2}
$$

where $Z p_{k}$ is the impedance of port $k$. According to (18), the reflection rate for port $k$ is

$$
\rho_{k}=-\frac{1}{1+\frac{2 Z p_{k}}{Z_{0}}} .
$$

Obviously, when $Z p_{k}$ is large compared to $Z_{0}, \xi_{k}$ is close to 1 and $\rho_{k}$ is close to 0 .

Without loss of generality, we assume that a signal propagates from port $p$ to $q$ and $p<q$. The signal passes through $q-p-1$ ports before reaching port $q$. According to (20), in addition to line attenuation, the received signal will be attenuated due to port reflections by an extra factor

$$
\eta_{p q}=\left\{\begin{array}{ll}
\prod_{k=p+1}^{q-1} \xi_{k} & q-p \geq 2 \\
1 & q-p=1
\end{array} .\right.
$$

Combining (19) and (22), the signal across the receiver input resistance $R_{r}$ after correction is

$$
V_{\text {sig }}=\eta_{p q} \frac{\frac{R_{r} Z_{0}}{2}}{\left(\frac{Z_{0}}{2}+Z_{s}\right)\left(\frac{Z_{0}}{2}+Z_{r}\right)} \exp \left(-\gamma \ell_{p q}\right) V_{s} .
$$

Because we require a small reflection rate for a large SNR, higher order reflections result in negligible noise. Therefore, to compute the reflection noise from ports, we only consider the first-order reflection. In this case, the reflected wave interfering with the signal at the receiver must come from those ports before the transmitter or after the receiver. Assuming there are $n$ ports in all, the reflection noise at the receiver port $q$ is

$$
\begin{aligned}
V_{\text {ref }}= & \sum_{k=q+1}^{n} \eta_{s k} \rho_{k} \eta_{r k} \frac{\frac{Z_{0}}{2}}{\frac{Z_{0}}{2}+Z_{s}} \exp \left(-\gamma\left(\ell_{s k}+\ell_{k r}\right)\right) V_{s} \\
& +\sum_{k=1}^{s-1} \eta_{s k} \rho_{k} \eta_{r k} \frac{\frac{Z_{0}}{2}}{\frac{Z_{0}}{2}+Z_{s}} \exp \left(-\gamma\left(\ell_{s k}+\ell_{k r}\right)\right) V_{s}
\end{aligned}
$$

We verified closed-form models (23) and (24) by comparing them with the solution derived from the accurate model in Section II-B. A large number of experiment settings are randomly generated and the results are shown in Fig. 5. Since in good designs we require small reflection rates for a large SNR, the maximum reflection rates are set to $15 \%$ in these experiments. From the figures, we can see that both (23) and (24) are highly accurate compared to the numerical solution in Section II-B. Especially in Fig. 5(b), in which we compare our noise model with the overall noise including the noise of all orders, the results of our model is very close to the overall noise with the average error of $6 \%$ and the maximum error of $18 \%$. This verifies our assumption of ignoring the higher-order reflection noise.

3) Effect of Terminations: According to (7), when the terminations are equal to the characteristic impedance $Z_{0}$, there will be no reflection from the terminations. Although perfect termination may be designed, the terminations may be different from the ideal case because of process variations. Imperfect terminations cause reflection and introduce extra noise. Similar to Section II-C2, the noise due to terminations is

$$
\begin{aligned}
V_{t}=\eta_{p n} \Gamma \eta_{q n} \frac{\frac{Z_{0}}{2}}{\frac{Z_{0}}{2}+Z_{s}} \exp \left(-\gamma\left(\ell_{p t 2}+\ell_{q t 2}\right)\right) V_{s} \\
+\eta_{1 p} \Gamma \eta_{1 q} \frac{\frac{Z_{0}}{2}}{\frac{Z_{0}}{2}+Z_{s}} \exp \left(-\gamma\left(\ell_{p t 1}+\ell_{q t 1}\right)\right) V_{s}
\end{aligned}
$$




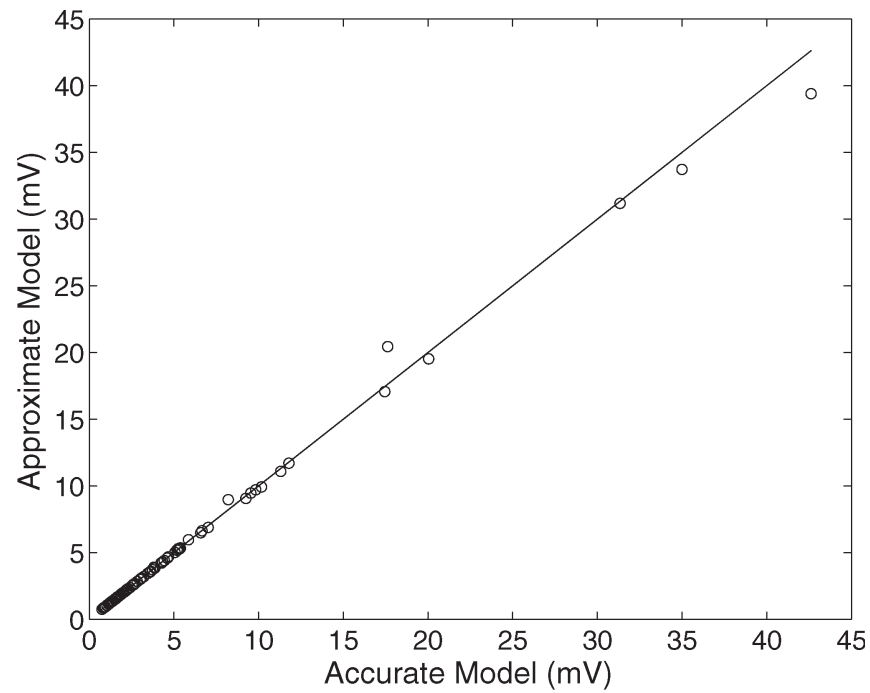

(a)

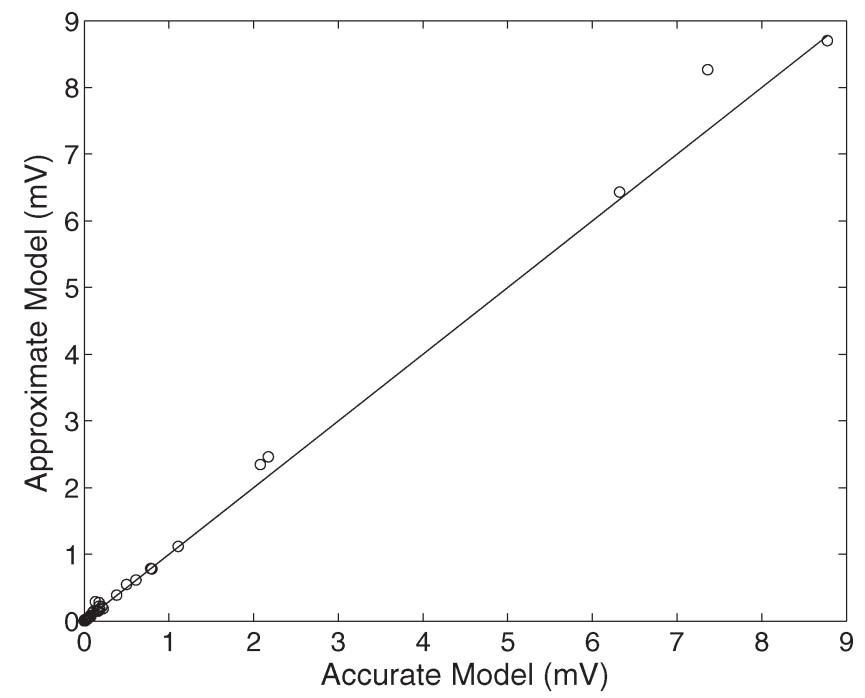

(b)

Fig. 5. Comparison between closed-form solution and numerical solution in Section II-B. (a) Equation (23) for signal at receivers. (b) Equation (24) for reflection noise from ports.

where $\ell_{p t 1}$ and $\ell_{p t 2}$ are the lengths from port $p$ to terminations 1 and 2 , respectively, and $\ell_{q t 1}$ and $\ell_{q t 2}$ are the lengths from port $q$ to terminations, respectively. To compute the upper bound of the noise, we assume $\eta$ close to 1 and the interconnect is low loss $(\alpha \approx 0)$. According to (23) and (25), the upper bound of reflection noise to signal ratio due to imperfect terminations is

$$
\zeta_{t}=2 \Gamma \text {. }
$$

Considering (6), we have

$$
\begin{aligned}
\frac{\partial \Gamma}{\partial Z_{t}} & =-\frac{2 Z_{0}}{\left(Z_{0}+Z_{t}\right)^{2}} \\
\Rightarrow \frac{\partial \Gamma}{\partial\left(\frac{Z_{t}}{Z_{0}}\right)} & =-0.5 .
\end{aligned}
$$

Therefore, if the maximum variation of the $Z_{t}$ is $15 \%$, the noise amplitude due to reflection can be up to $15 \%$ of the signal amplitude and the SNR is $16 \mathrm{~dB}$.

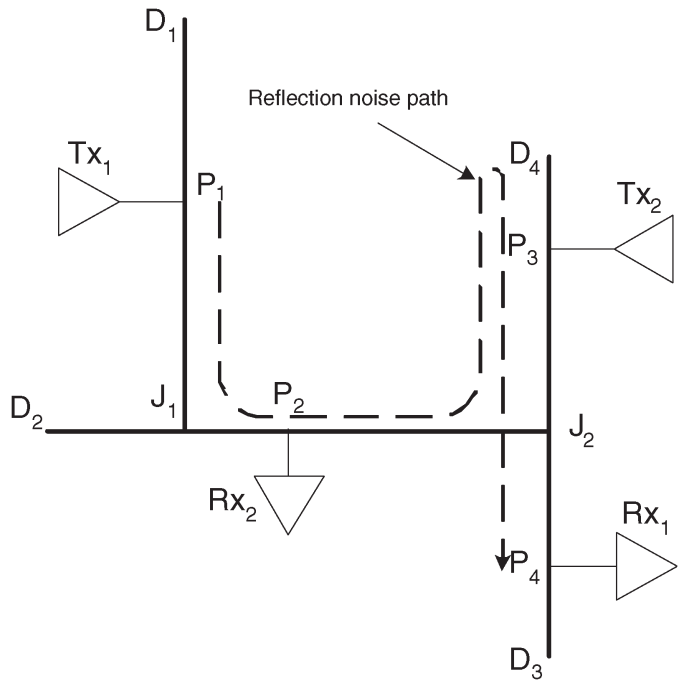

Fig. 6. Reflection path.

With imperfect terminations, the total noise due to port and termination reflections across the receiver input resistance is

$$
V_{n}=\frac{R_{r}}{Z_{r}}\left(V_{\text {ref }}+V_{t}\right)
$$

where $V_{\text {ref }}$ and $V_{t}$ are defined in (24) and (25), respectively. With perfect terminations, $V_{t=0}$. The signal at the receiver node is given in (23). The SNR at a receiver can be computed as

$$
\mathrm{SNR}=10 \log \frac{\frac{V_{\mathrm{sig}}^{2}}{2 R_{r}}}{\frac{V_{n}^{2}}{2 R_{r}}+P_{n}}
$$

where $P_{n}$ is the power of the intrinsic receiver noise. Note that when the terminations are perfect, $V_{t}$ in (29) is equal to zero, and (30) is the SNR with perfect terminations.

\section{SNR Model for Branched Transmission Line}

In this section, we further extend our model to consider branched RF interconnects. A branched RF interconnect has junctions or branching points connecting two or more uniform RF interconnects. These junctions introduce extra discontinuity to the RF signal and cause more loss of signal and reflection noise. Fig. 6 shows one example of branched RF interconnects. In this example, there are two channels. One channel connects transmitter $T x_{1}$ and receiver $R x_{1}$, and the other connects $T x_{2}$ and $R x_{2}$. The transceivers connect to ports $P_{1}$ to $P_{4}$. There are two junction points $J_{1}$ and $J_{2}$, and the RF interconnect is terminated at terminations $D_{1}$ to $D_{4}$. To study the SNR at receivers, all the discontinuities including ports, junctions, and terminations must be considered. Furthermore, in a branched $\mathrm{RF}$ interconnect, noise can be reflected from all the branches to affect a receiver. For example, in Fig. 6, noise reflected from $D_{4}$ can affect the signal at $R x_{1}$ following the path illustrated in the figure. A possible path for reflection noise is defined as a reflection path. In this study, we normally consider all the paths for first-order reflection, which has only one reflection in the 


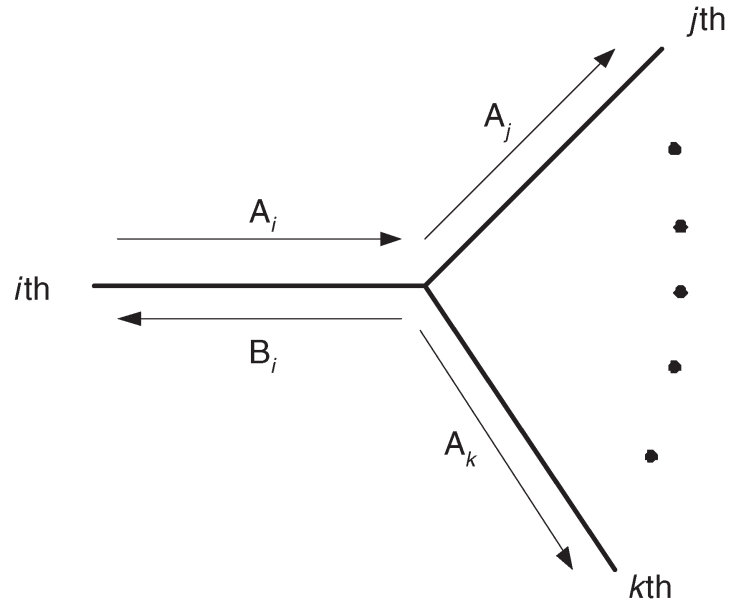

Fig. 7. Signals at a branch point.

entire path. This assumption is reasonable because we require the high SNR and noise is much smaller than the signal.

The reflection and transmission rates of ports and terminations can be computed in the same way as in single branch case in Section II-C. To consider the effects of junctions, we need to compute the signal transmission rate and reflection rate of each branch. Considering a junction connecting $n$ branches, let the characteristic impedance of the $i$ th branch connected to the junction be $Z_{0 i}$. For a signal traveling on the $i$ th branch toward the junction, part of the signal will be reflected due to the discontinuity and the rest will be transmitted to other branches. Since we will consider reflections from other the discontinuity separately and temporarily ignore them, the signals on all the branches are shown in Fig. 7. Using KCL and KVL, the reflection rate for branch $i$ is derived as

$$
\rho_{i}=\frac{Z_{t i}-Z_{0 i}}{Z_{t i}+Z_{0 i}}
$$

where

$$
Z_{t i}=\frac{1}{\sum_{j \neq i} \frac{1}{Z_{0 j}}}
$$

The transmission rate is

$$
\xi_{i}=\frac{2 Z_{t i}}{Z_{t i}+Z_{0 i}}
$$

With the transmission and reflection rates of the discontinuities, including ports, junctions, and terminations, the signal received by receiver $r$ from transmitter $s$ is derived as

$$
V_{s i g}=k_{s, r} \prod_{i \in s \rightarrow r, i \neq s} \exp \left(-l_{i-1, i} \gamma_{i-1, i}\right) \xi_{i} \cdot V_{s}
$$

where $s \rightarrow r$ is the shortest path from $s$ to $r . l_{i-1, i}$ is the branch length between the $(i-1)$ th and $i$ th discontinuities, $\gamma_{i-1, i}$ is the propagation constant of the branch, and $\xi_{i}$ is the $i$ th discontinuity's transmission rate. $k_{s, r}$ is a coefficient depending on the transmitter and receiver, and is defined as

$$
k_{s, r}=\frac{\frac{R_{r} Z_{0 r}}{2}}{\left(\frac{Z_{0 s}}{2}+Z_{s}\right)\left(\frac{Z_{0 r}}{2}+Z_{r}\right)}
$$

where $R_{r}$ is the receiver input resistance, and $Z_{0 s}$ and $Z_{0 r}$ are the characteristic impedance of the branches where the transmitter and the receiver are located, respectively.

The first-order reflection noise at the receiver $r$ from transmitter $s$ is

$$
\begin{aligned}
V_{n, 1}=k_{s, r} & \sum_{p}\left(\prod_{i \in s \rightarrow p, i \neq s} \exp \left(-l_{i-1, i} \gamma_{i-1, i}\right) \xi_{i}\right. \\
& \left.\times \rho_{p} \prod_{j \in p \rightarrow r, j \neq s} \exp \left(-l_{j-1, j} \gamma_{j-1, j}\right) \xi_{j}\right) \cdot V_{s}
\end{aligned}
$$

where port $p$ is a port not on the shortest path from $s$ to $r$, and $\rho_{p}$ is the reflection rate of the $p$ th discontinuity. The SNR therefore can be readily computed from (34) and (36).

\section{E. Frequency Dependence of Attenuation and Phase Delay}

Distortion of the waveform depends on attenuation and phase delay. Attenuation is defined as the reduction of the signal amplitude compared to the original signal. The phase delay is defined as

$$
P(\omega)=-\frac{\Delta \phi(\omega)}{\omega}
$$

where $\Delta \phi$ is the frequency dependent phase changing compared to the original signal and $\omega$ is the radial frequency of the carrier. A distortionless communication channel should have attenuation and phase delay, both uniform over the frequency band for a frequency channel.

To ensure small distortion, we require limited difference of phase delay and attenuation in a frequency channel

$$
\begin{gathered}
\Delta P=\frac{\left|P\left(\omega_{0}-\omega_{b}\right)-P(\omega)\right|}{T_{b}}<0.01 \\
\Delta M=\frac{\left|M\left(\omega_{0}-\omega_{b}\right)-M\left(\omega_{0}\right)\right|}{M\left(\omega_{0}\right)}<0.01
\end{gathered}
$$

where $\omega_{0}$ is the carrier frequency, $\omega_{b}$ is the digital baseband frequency, and $T_{b}$ is the baseband period. Phase $P$ and amplitude $M$ are computed according to (23); (38) and (39) are our FOM for signal distortion.

\section{Synthesis OF MultibAnd CPW RF InTERCONNECT}

In this section, we apply the models developed in Section II to optimize the area of CPW-typed RF interconnect with multiple transceivers and multiple frequency bands. We first review multiband RF interconnects and then discuss computation of 


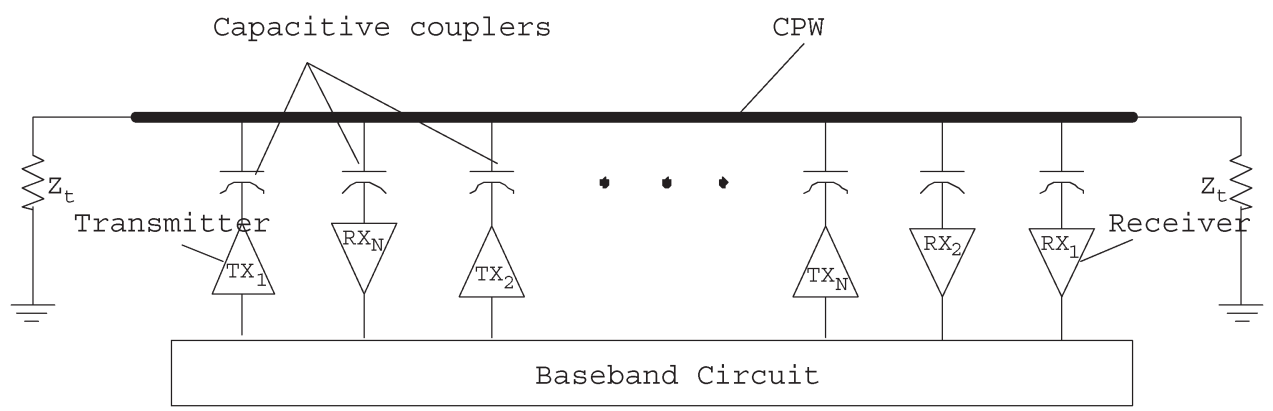

Fig. 8. On-chip RF interconnect structure.

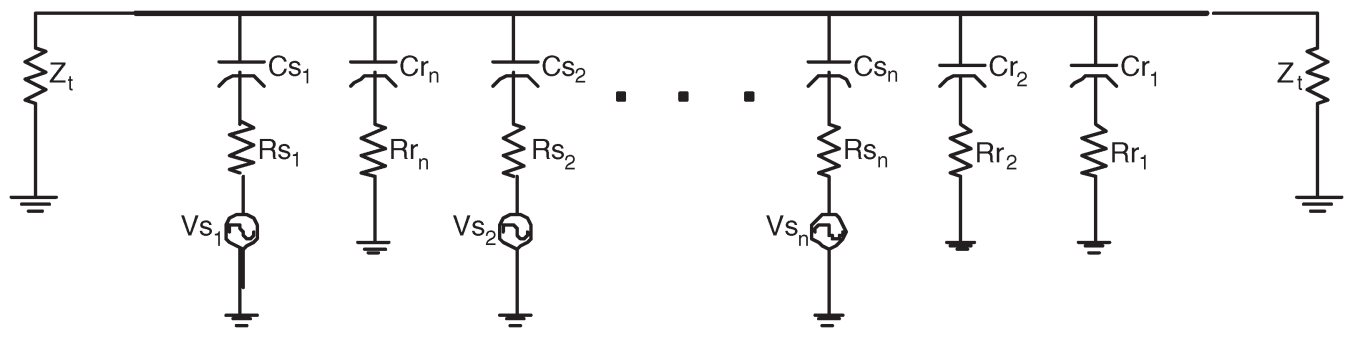

Fig. 9. Circuit model of RF interconnect.

the transmission line parameters and circuit model. Finally, we present the synthesis of RF interconnects with simulated annealing method.

\section{A. Review of Multiband RF Interconnects}

As shown in Fig. 8 for on-chip RF interconnect, the digital signal is first mixed with an RF carrier by a transmitter and then coupled via a capacitive coupler into the interconnect, which is a transmission medium such as a CPW or a microstrip transmission line (MTL). The RF signal is transmitted bidirectionally along the interconnect and picked up by multiple receivers via capacitive couplers and demodulated to obtain the original digital signal. Compared to the traditional interconnect, the RF interconnect has several advantages, such as low loss and distortion [10], immunity to digital switching noise [15], and less switching noise [15]. More importantly, a single RF interconnect can be simultaneously shared by several communication channels with multiaccess techniques such as FDMA and CDMA. By allocating frequency bands, FDMA allows multiple channels operated at different frequencies simultaneously access the interconnect without interfering with each other. CDMA further allows each frequency channel to be divided into subchannels by different encodings. With multiaccess schemes, one RF interconnect can transmit multiple digital signals simultaneously; thus, the bus structure can be avoided and area can be saved. In addition, the communication channel can be reconfigured in real time by changing the spreading codes.

Similar to other analog communication connections, the performance of an RF interconnect is limited by the bit error rate (BER) at the receiver. To achieve the required BER, its SNR should be larger than a minimum bound [10] and the signal distortion should be controlled in certain range. Low distortion requires close to uniform attenuation and phase delay in the band interested in. Because the signal experiences loss and distortion through both the capacitive coupler and the interconnect, the size of the coupler and the geometries of RF interconnects must be designed properly for acceptable degree of loss and distortion.

\section{B. Circuit Model of CPW Interconnect}

For CPW, we denote the signal wire width as $w$, the shielding wire width as $g$, and spacing between signal and shielding wires as $s$. The transmission line parameters can be computed from partial wire parameters as [5]

$$
\begin{aligned}
& R=R_{s}+\frac{R_{g}}{2} \\
& L=L_{s}-2 L_{s g}+\frac{L_{g g}}{2}+\frac{L_{g}}{2} \\
& C=2 C_{s g}+C_{s}
\end{aligned}
$$

where $R_{s}, C_{s}$, and $L_{s}$ are the self resistance, ground capacitance, and partial self inductance of the signal wire. $R_{g}$ and $L_{g}$ are the self resistance and partial inductance of the shielding wires. $C_{s g}$ and $L_{s g}$ are the coupling capacitance and partial mutual inductance between the signal wire and a shielding wire. $L_{s s}$ is the partial mutual inductance between the two shielding wires. We extract frequency-dependent resistance and partial inductance with FastHenry [31]. Because we assume uniform dielectric, according to [30], [32], and [33] calculate the capacitance by

$$
C=\frac{1}{c^{2} L}
$$

where uniform dielectric is assumed and $c$ is the speed of light in the dielectric. The capacitive couplers of transceivers are modeled by lumped capacitors, and the transceivers are modeled by linear drivers. The circuit model of the entire RF interconnect is shown in Fig. 9. 


\section{Automatic Synthesis}

We assume that the transceivers have been given and the noise characteristics of the receivers are determined beforehand. We minimize the area under the constraints of SNR and distortion by adjusting the geometries of the interconnect and the size of each capacitive coupler. SNR must be larger than a minimum value. For small distortion, we require the solutions satisfy FOM (38) and (39) for signal distortion. The problem is formulated as below.

Formulation 1: Given the transceivers and the noise characteristics of receivers $P_{n}$ [see (30)], determine the signal wire width $w$, signal shield spacing $s$, shielding width $g$, and the size of each capacitive coupler such that the total area of the interconnect and capacitive couplers is minimized under the constraint that at each receiver our FOMs (38) and (39) for signal distortion are satisfied and SNR is larger than the required minimum SNR.

Our closed-form models in (30), (38), and (39) enable us to use simulated annealing $(S A)$ algorithm to optimize the wire geometries and the size of each capacitive coupler in a short runtime. The objective function is defined as

$$
F\left(w_{j}, s_{j}, g_{j}, C_{i}\right)=K_{a} A+\sum_{i}\left(K_{s} \mathrm{FS}_{i}+K_{p d} \mathrm{FP}_{i}+K_{a d} \mathrm{FA}_{i}\right)
$$

where $K_{a}, K_{s}, K_{p d}$, and $K_{a d}$ are weights, and $A$ is the total area of the RF interconnect and couplers. $w_{j}, s_{j}$, and $g_{j}$ are the signal wire width, signal to shield spacing, and shield width of branch $j$, and $C_{i}$ s are the sizes of capacitive couplers. We also require that the couplers totally overlap the central signal wire of CPW and do not overlap each other. We assume that the coupler can be implemented with a capacitance density of $0.5 \mathrm{fF} / \mu \mathrm{m}^{2}[14]$. Then

$$
A=\sum_{j=1}^{b} l_{j}\left(w_{j}+2 s_{j}+2 g_{j}\right)+\sum_{i=1}^{n} \frac{C_{i}}{0.5} .
$$

$\mathrm{FS}_{i}, \mathrm{FP}_{i}$, and $\mathrm{FA}_{i}$ in (44) are the penalty functions for the violation of the constraints of SNR, phase delay, and attenuation at receiver $i$. For each receiver

$$
\mathrm{FS}_{i}= \begin{cases}0, & \left(\mathrm{SNR}_{i}>\underline{\mathrm{SNR}}\right) \\ \underline{\mathrm{SNR}}-\mathrm{SNR}_{i}, & \left(\mathrm{SNR}_{i}<\underline{\mathrm{SNR}}\right)\end{cases}
$$

where $\underline{\mathrm{SNR}}$ is the minimum SNR

$$
\mathrm{FP}_{i}= \begin{cases}0, & \left(\Delta P_{i}<\overline{\Delta P_{i}}\right) \\ \Delta P_{i}-\overline{\Delta P_{i}}, & \left(\Delta P_{i}>\overline{\Delta P_{i}}\right)\end{cases}
$$

where $\Delta P_{i}$ is defined in (38) and $\overline{\Delta P_{i}}$ is the upper bound of the phase delay difference. Similarly

$$
\mathrm{FA}_{i}= \begin{cases}0, & \left(\Delta M_{i}<\overline{\Delta M_{i}}\right) \\ \Delta M_{i}-\overline{\Delta M_{i}}, & \left(\Delta M_{i}>\overline{\Delta M_{i}}\right)\end{cases}
$$

where $\Delta M_{i}$ is defined in (39) and $\overline{\Delta M_{i}}$ is an upper bound FOM of attenuation difference.
TABLE I

Comparison Between Manual Design And Automatic Synthesis

\begin{tabular}{|c|c|c|c|c|c|c|}
\hline design & $\mathrm{w}$ & $\mathrm{s}$ & $\mathrm{g}$ & total width & $C_{s}$ & $C_{r}$ \\
\hline manual & - & - & - & $100 \mu \mathrm{m}$ & $100 \mathrm{fF}$ & $100 \mathrm{fF}$ \\
\hline synthesis & $2.2 \mu \mathrm{m}$ & $6.0 \mu \mathrm{m}$ & $1.1 \mu \mathrm{m}$ & $16.8 \mu \mathrm{m}$ & $51 \mathrm{fF}$ & $49 \mathrm{fF}$ \\
\hline
\end{tabular}

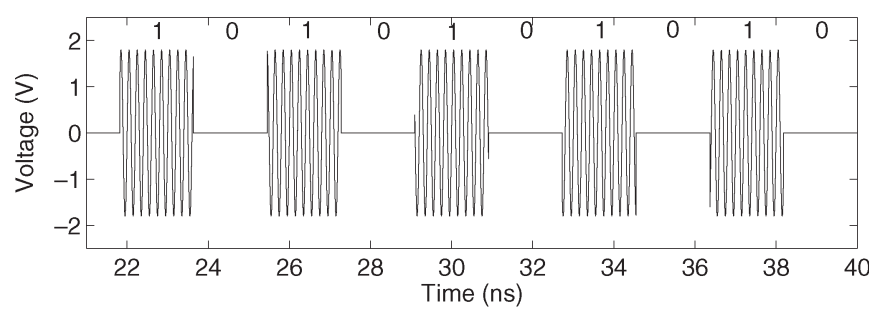

(a)

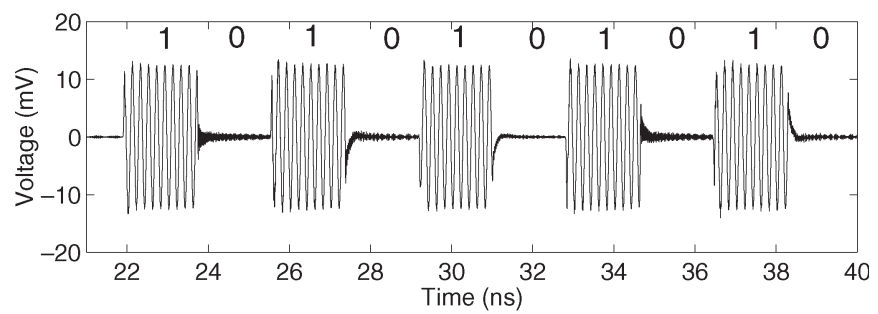

(b)

Fig. 10. Transient waveform. (a) Input. (b) Output.

There are four types of moves in our simulated annealing scheme: 1) changing $w_{j} ; 2$ ) changing $g_{j} ; 3$ ) changing $s_{j}$; and 4) changing $C_{i}$. Branch $i$ and coupler $j$ are randomly picked. During the process, we always make sure that the coupler fully overlapped with the transmission line. In each movement, we randomly increase or decrease the chosen geometric parameter by a factor from $0 \%$ to $5 \%$. We start the SA with initial temperature of 20 and terminate it at 0.001 . The temperature is decreased by a factor of 0.95 and the number of moves at a particular temperature is 300 .

\section{Synthesis Results for Single RF Interconnect}

1) Perfect Terminations: In this section, we assume the terminations are perfect and therefore there is no reflection from terminations. For comparison, we first synthesize an RF interconnect with the same specifications as the manual design case in [10]. The interconnect length is $1 \mathrm{~cm}$, the carrier frequency is $5 \mathrm{GHz}$, the baseband frequency is $275 \mathrm{MHz}$, the transceiver impedance is $2 \mathrm{k} \Omega$, the transmitter voltage is $1.8 \mathrm{~V}$, and the power of receiver intrinsic noise is $-67 \mathrm{dBm}$. The minimum SNR is set to $20 \mathrm{~dB}$. One transmitter and one receiver are at the opposite ends of the interconnect. As shown in Table I, the synthesis result reduces the total interconnect width including spacing by $80 \%$ and coupler size by $50 \%$ compared to the manual design in [10]. To verify our design, we carry out transient simulation with SPICE. Linear transceivers are assumed in the simulation. The input digital signal pattern is alternative "0" and "1." Fig. 10 plots the input and output waveforms with clear repeated "01" pattern. To compute the SNR of the synthesized interconnects, we use SPICE to measure the frequency response $V_{e}$ at the carrier frequency and then 
TABLE II

Automatic Synthesis Results. All Results Meet SNR and Distortion Requirements

\begin{tabular}{|c|c|c|c|c|c|c|c|c|c|c|}
\hline & \multicolumn{4}{|c|}{ system specifications } & \multicolumn{6}{|c|}{ synthesis result } \\
\hline case & ports & channels & $R p(\Omega)$ & $l(\mathrm{~cm})$ & $w(\mu m)$ & $s(\mu m)$ & $g(\mu m)$ & $\operatorname{Area}\left(\mu m^{2}\right)$ & Avg. $C_{s}(f F)$ & Avg. $C_{r}(f F)$ \\
\hline 1 & 2 & 1 & 500 & 1 & 1.6 & 0.8 & 1.2 & 56360 & 88 & 92 \\
\hline 2 & 2 & 1 & 1000 & 1 & 1.8 & 2.3 & 1.1 & 85916 & 95 & 98 \\
\hline 3 & 2 & 1 & 2000 & 1 & 2.3 & 8.6 & 1.9 & 260894 & 67 & 59 \\
\hline 4 & 2 & 1 & 1000 & 2 & 2.7 & 3.3 & 1.9 & 279476 & 103 & 103 \\
\hline 5 & 2 & 1 & 1000 & 3 & 4.3 & 3.5 & 3.0 & 517773 & 108 & 113 \\
\hline 6 & 40 & 5 & 500 & 1 & 3.6 & 0.9 & 2.6 & 109532 & 55 & 28 \\
\hline 7 & 40 & 5 & 1000 & 1 & 4.5 & 4.5 & 3.0 & 196305 & 40 & 19 \\
\hline
\end{tabular}

compute the signal $V_{s}$ according to (34). The reflection noise is derived as $V_{n}=V_{e}-V_{s}$. Because we assume that the intrinsic noise is given, the SNR can be readily computed by (30). In this experiment, the SNR is $21 \mathrm{~dB}$, which satisfies the lower bound constraint of $20 \mathrm{~dB}$.

We also carried out synthesis with different transceiver impedance and various numbers of FDMA channels and transceivers. The FDMA channels are allocated from 10 to $110 \mathrm{GHz}$ with $20 \mathrm{GHz}$ for each channel. When there is only one FDMA channel, a 10- to 30-GHz channel is used. The digital baseband frequency is $1 \mathrm{GHz}$. The transmitter voltage amplitude, the receiver amplifier noise, and the minimum SNR are the same as those in the experiment presented above. The transmitters and receivers have the same impedance and are uniformly distributed along the interconnect. Table II summarizes some sample results. In this table, we report the average capacitive coupler size for transmitters and receivers. From the results, it is clear that the synthesized solution for different input parameters varies greatly. The total area can be $3 \times$ different. Generally, larger transceiver impedance, longer communication distance, and more ports lead to wider interconnect and larger area. Note the manual design assumes uniform capacitive coupler size for all ports, but we find that the coupler size can vary a lot depending on the channel, location, and type of each port. The difference can be up to $20 \times$ in the same design case. More interestingly, when there are multiple ports, the capacitive couplers for receivers are much smaller than those for transmitters, which helps reduce reflection and increase the signal transmission rate.

To show the effectiveness of the synthesis, we also carried out transient simulations with different interconnect settings. In Fig. 11(a) shows the waveform at port 40 of case 7 in Table II with the matched interconnect from synthesis and (b) shows the waveform with mismatched interconnect synthesized for case 1 . It is clear that (a) satisfies the SNR constraints while (b) has only $2 \mathrm{mV}$ of signal amplitude and an SNR of $7 \mathrm{~dB}$, which is far below the required bound.

2) Imperfect Terminations: In reality, the terminations are often mismatched due to process variations. In this section, we study the impact of imperfect terminations on the synthesis results. The RF interconnect under study is $1 \mathrm{~cm}$ long with five channels allocated from 10 to $110 \mathrm{GHz}$ with $20 \mathrm{GHz}$ for each channel. Each channel has one transmitter and four receivers. The locations of the ports are randomly selected. The lower bound of SNR is set to $15 \mathrm{~dB}$. We define the mismatch degree as the relative difference between the real termination and the

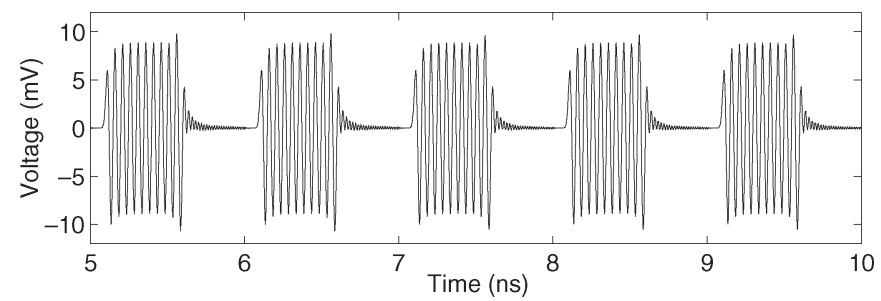

(a)

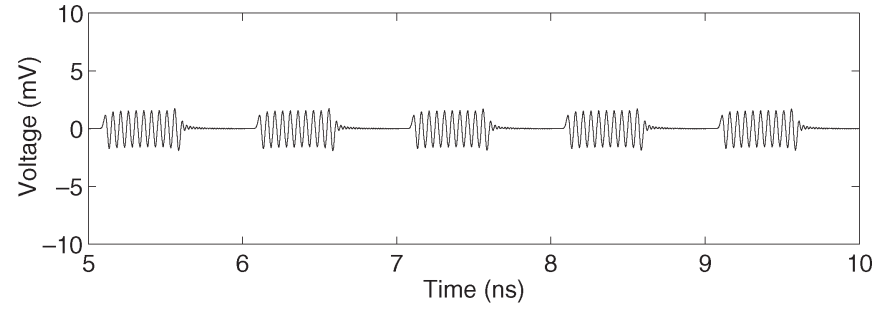

(b)

Fig. 11. Transient waveforms of interconnect configuration case 7 in Table II (a) With matched interconnect. (b) With mismatched interconnect synthesized for case 1 .

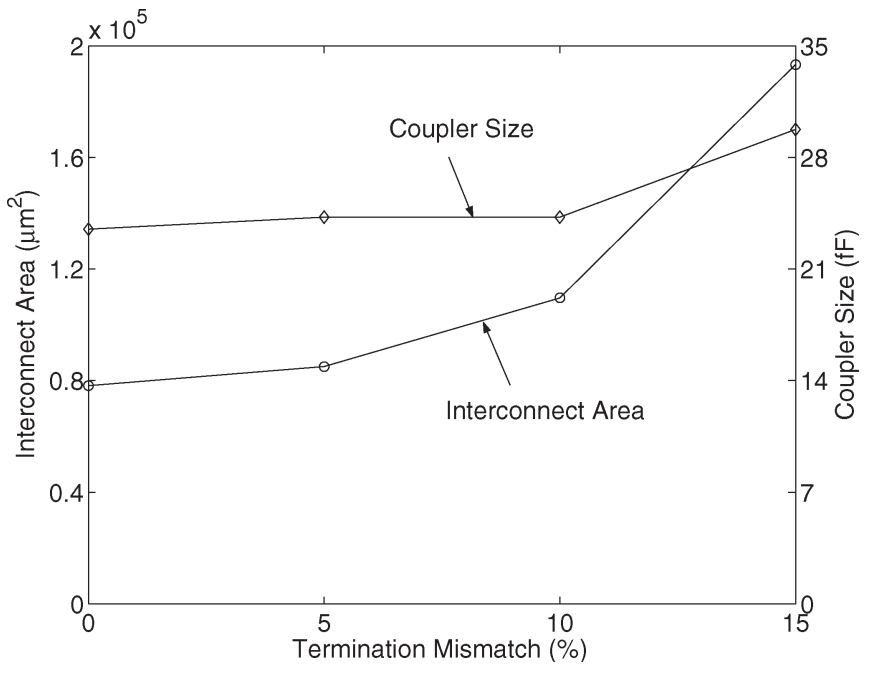

Fig. 12. Interconnect area and coupler size with different termination mismatch. The solutions meet the SNR and distortion requirements.

perfect termination. Fig. 12 shows the trend of the interconnect area and coupler size with the increase of different degrees of mismatch. It is clear the total interconnect area increases with the increase of mismatch. Initially, the area increases slowly and the mismatch has less effects on the synthesis results when the mismatch is less than $10 \%$. However, when the mismatch 


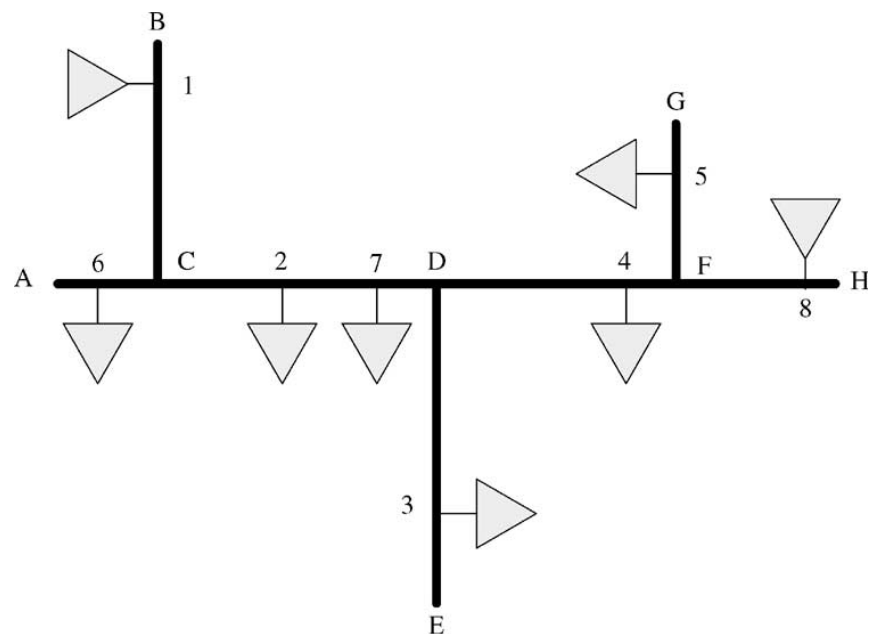

Fig. 13. Sample structure of branched RF interconnects.

gets close to $15 \%$, the area increases dramatically to about three times of the area of perfect matching case. The coupler size has similar trends but with much smaller slope. According to the previous discussion in Section II-C3, the SNR with only reflection noise from the terminations decreases to $16 \mathrm{~dB}$ when the mismatch is $15 \%$. When the mismatch is larger than $15 \%$, no valid solutions are found.

\section{E. Synthesis Results for Branched RF Interconnect}

In this section, we apply the SNR and signal distortion models to synthesize an RF interconnect with branches. In this experiment, the impedances of all the transceivers are $1000 \Omega$ and we assume perfect terminations. The minimum SNR is set to $15 \mathrm{~dB}$. The transmitter input voltage is $1.8 \mathrm{~V}$. In Fig. 13, we show one example of branched RF interconnects. The structure has one main interconnect branch and three subbranches. However, since there is already discontinuity at branching points, we allow each segment between branching points in the main branch have different geometries to further optimize the structure. Therefore, segments AC, CD, DF, and FH can have different optimal geometries. All these branches are CPW. There are two channels and each of them has one transmitter. Channel 1 is at a frequency of $10 \mathrm{GHz}$ and has four receivers at ports 2, 3, 4, and 5 receiving signal from the transmitter at port 1. Channel 2 is at frequency of $20 \mathrm{GHz}$ and has two receivers at ports 6 and 7 receiving signal from the transmitter at port 8. Table III shows the synthesized geometries of each segment and Table IV shows the synthesized value of the coupling capacitor at each port. From the results, we can see the synthesized values for each segment are different. For segments shared by signal paths such as CD and DF, signal wire width and shield width are large to reduce the attenuation of the signal. Subbranch segments have smaller signal width and shield width, but the spacing between them can be large to match the impedance at the branching points. For each channel, the transmitter has the largest coupling capacitor compared to receivers, and receivers farther from the transmitters have larger coupling capacitors than those closer to the transmitters.
TABLE III

SyNTHESIZED GEOMETRIES OF EACH CPW SEGMENT

\begin{tabular}{|c|c|c|c|c|c|}
\hline branch & length $(\mu m)$ & $\mathrm{w}(\mu \mathrm{m})$ & $\mathrm{s}(\mu \mathrm{m})$ & $\mathrm{g}(\mu \mathrm{m})$ & area $\left(\mu m^{2}\right)$ \\
\hline $\mathrm{AC}$ & 1000 & 0.5 & 5.1 & 0.1 & 10900 \\
$\mathrm{BC}$ & 5000 & 2.7 & 6.1 & 1.5 & 89500 \\
$\mathrm{CD}$ & 5000 & 5.8 & 2.3 & 2.3 & 75000 \\
$\mathrm{DE}$ & 6000 & 1.8 & 6.1 & 0.9 & 94800 \\
$\mathrm{DF}$ & 4000 & 6.4 & 3.4 & 2.2 & 70400 \\
$\mathrm{FG}$ & 3000 & 0.8 & 8.0 & 0.3 & 52200 \\
$\mathrm{FH}$ & 2000 & 2.3 & 10 & 1.1 & 90400 \\
\hline
\end{tabular}

TABLE IV

SYNTHESIZED COUPLING CAPACITANCE FOR PORTS

\begin{tabular}{|c|c|c|c|c|c|c|c|c|}
\hline Port & 1 & 2 & 3 & 4 & 5 & 6 & 7 & 8 \\
\hline Coupling cap. (fF) & 67 & 13 & 47 & 32 & 58 & 12 & 21 & 48 \\
SPICE (mV) & - & 14.8 & 8.9 & 9.9 & 8.7 & 14.5 & 9.0 & - \\
Model (mV) & - & 14.6 & 8.7 & 10.3 & 8.2 & 13.4 & 8.7 & - \\
\hline
\end{tabular}

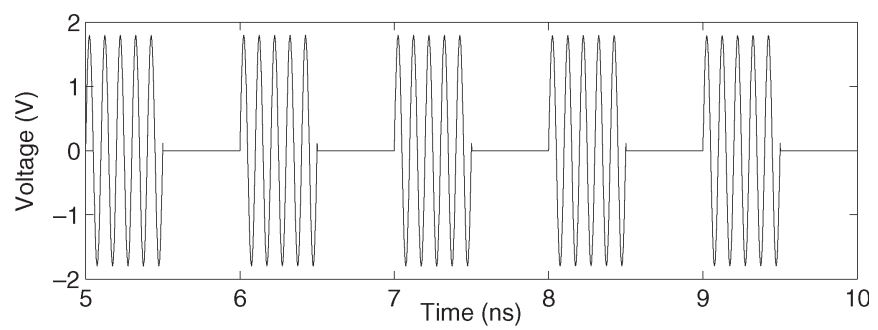

(a)

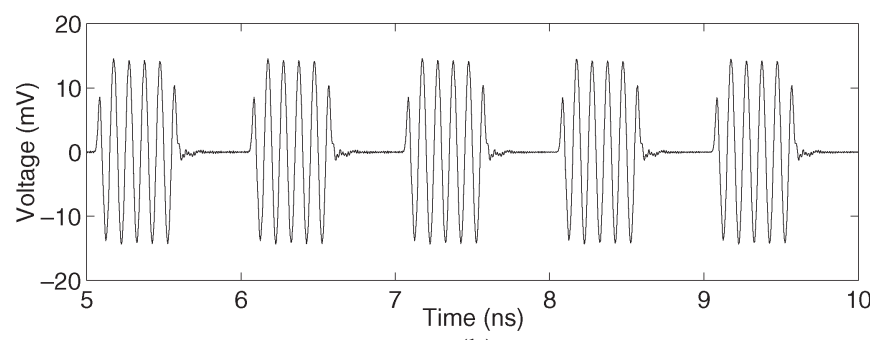

(b)

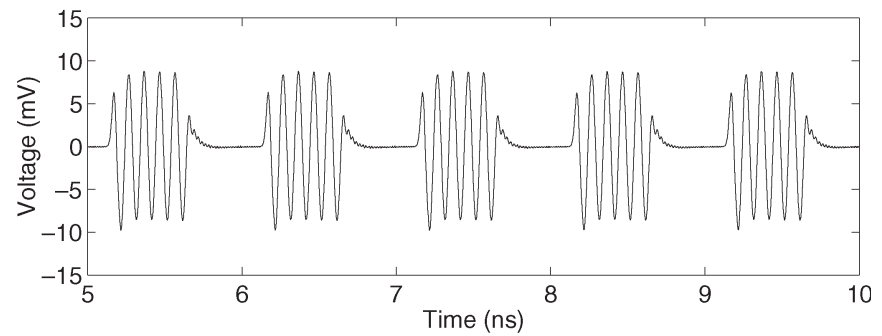

(c)

Fig. 14. Transient waveforms at various ports. (a) Port 1 (transmitter). (b) Port 2. (c) Port 5.

We further carry out SPICE simulation to verify the results. The amplitudes of signals at each receiver from both SPICE simulation and the proposed model are shown in Table IV. We can see that the results of our model closely match those from SPICE simulation. In Fig. 14, we show the waveforms at transmitter 1 and receivers 2 and 5 from SPICE simulation. Port 5 is farthest from port 1 and its signal amplitude is just enough to meet the $15-\mathrm{dB}$ minimum requirement. The signal amplitude at port 2 is slightly larger because it is closer to the transmitter. 


\section{CONCLUSION AND DisCUSSION}

Multichannel multiport interconnects that transmit signals via high-frequency carriers have been used for high-speed high-bandwidth intrachip and interchip communications. To efficiently analyze and design such interconnection, we first developed an efficient model with linear complexity to compute the voltages of a multiport transmission line and then derived closed-form models for SNR at receivers. We also proposed FOMs to minimize the distortion in signal phase and amplitude. Experiments show that the SNR model is accurate compared to SPICE simulation and the signal distortion FOMs are effective. We applied our models to the automatic synthesis of CPW geometries and capacitive couplers for branched multichannel multiport RF interconnection. We minimized the total interconnect area under the constraints of SNR and signal distortion. The solutions are verified with time-domain transient simulations. Compared to the published manual designs, the synthesized solutions can save up to $80 \%$ chip area. The complexity and large difference in the various optimized solutions demonstrate the necessity and effectiveness of our models and the automatic synthesis process. For future work, we plan to extend our models to further consider active devices and apply these models to other types of transmission-line-based interconnect designs.

\section{REFERENCES}

[1] R. T. Chang, N. Talwalkar, C. P. Yue, and S. S. Wong, "Near speed-oflight signaling over on-chip electrical interconnects," IEEE J. Solid-State Circuits, vol. 38, no. 5, pp. 834-838, May 2003.

[2] ITRS, International Technology Roadmap for Semiconductors (Semiconductor and Industry and Association), (2003). [Online]. Available: http://public.itrs.net

[3] M. Minzuno, K. Anjo, Y. Sumi, M. Fukaishi, H. Wakabayashi, T. Mogami, T. Horiuchi, and M. Yamashina, "Clock distribution networks with on-chip transmission lines," in Proc. Int. Interconnect Technology Conf., Burlingame, CA, 2000, pp. 3-5.

[4] J. D. Warnock, J. M. Keaty, J. Petrovick, J. G. Clabes, C. J. Kircher, B. L. Krauter, P. J. Restle, B. A. Zoric, and C. J. Anderson, "The circuit and physical design of the POWER4 microprocessor," IBM J. Res. Develop., vol. 46, no. 1, pp. 27-51, 2002.

[5] R. Escovar and R. Suaya, "Transmission line design of clock trees," in Proc. ICCAD, San Jose, CA, 2002, pp. 334-340.

[6] B. M. Beckmann and D. A. Wood, "TLC: Transmission line caches," in Proc. Int. Symp. Microarchitecture, San Diego, CA, 2003, pp. 43-54.

[7] P. Wang, G. Pei, and E.-C. Kan, "Pulsed wave interconnect," IEEE Trans. Very Large Scale Integr. (VLSI) Syst., vol. 12, no. 5, pp. 453-463, May 2004

[8] W. Ryu, J. Lee, H. Kim, S. Ahn, N. Kim, B. Choi, D. Kam, and J. Kim, "RF interconnect for multi-Gb/s board-level clock distribution," IEEE Trans. Adv. Packag., vol. 23, no. 3, pp. 398-407, Aug. 2000

[9] W. Ryu, A. L. C. Wai, F. Wei, W. Lai, and J. Kim, "Over GHz low-power $\mathrm{RF}$ clock distribution for a multiprocessor digital system," IEEE Trans. Adv. Packag., vol. 25, no. 1, pp. 18-27, Feb. 2002.

[10] M. F. Chang, V. P. Roychowdhury, L. Zhang, H. Shin, and Y. Qian, "RF/wireless interconnect for inter- and intra-chip communications," Proc. IEEE, vol. 89, no. 4, pp. 456-466, Apr. 2001.

[11] V. Chi, "Salphasic distribution of clock signals for synchronous systems," IEEE Trans. Comput., vol. 43, no. 5, pp. 597-602, May 1994.

[12] J. Wood, T. Edwards, and S. Lipa, "Rotary traveling-wave oscillator arrays: A new clock technology," IEEE J. Solid-State Circuits, vol. 36, no. 11, pp. 1654-1665, Nov. 2001.

[13] F. O'Mahony, C. Yue, M. Horowitz, and S. Wong, "A 10-GHz global clock distribution using coupled standing-wave oscillators," IEEE J. Solid-State Circuits, vol. 38, no. 11, pp. 13-21, Nov. 2003.

[14] H. Shin and M. F. Chang, "1.1 Gb/s RF-interconnect based on $10 \mathrm{GHz}$ RF-modulation in $0.18 \mu \mathrm{m}$ CMOS," Electron. Lett., vol. 38, no. 2, pp. 71-72, Jan. 2002.
[15] H. Shin, Z. Xu, K. Miyashiro, and M. F. Chang, "Estimation of signalto-noise ratio improvement in RF-interconnect," Electron. Lett., vol. 38 , no. 25 , pp. $1666-1667$, Dec. 2002

[16] H. Shin, Z. Xu, and M. F. Chang, "RF-interconnect for multi-Gb/s digital interface based on $10 \mathrm{GHz}$ RF-modulation in $0.18 \mu \mathrm{m}$ CMOS," in Proc. Microwave Symp. Dig., Seattle, WA, 2002, vol. 1, pp. 477-480.

[17] Q. Gu, Z. Xu, J. Kim, J. Ko, and M. F. Chang, "Three-dimensional circuit integration based on self-synchronized RF-interconnect using capacitive coupling," in Proc. Symp. VLSI Technology, Honolulu, HI, 2004, pp. $96-97$.

[18] Q. Gu, Z. Xu, J. Ko, S. Hsien, and M. Chang, "A self-synchronized RF-interconnect for 3-dimensional integrated circuits," in Proc. IEEE Int. Symp. Circuits and Systems, Vancouver, BC, Canada, 2004, pp. IV-317-IV-320.

[19] M. F. Chang, V. Roychowdhury, L. Y. Zhang, S. N. Zhou, Z. Y. Wang, Y. C. W. P. X. Ma, C. S. Lin, and Z. J. Kang, "Multi-I/O and reconfigurable $\mathrm{RF} /$ wireless interconnect based on near field capacitive coupling and multiple access techniques," in Proc. IEEE Int. Interconnect Technology Conf., Burlingame, CA, 2000, pp. 21-22.

[20] F. Y. Chang, "Waveform relaxation analysis of RLCG transmission lines," IEEE Trans. Circuits Syst., vol. 37, no. 11, pp. 1394-1415, Nov. 1990.

[21] J. Roychowdhury, A. R. Newton, and D. O. Pederson, "An impulseresponse based linear time-complexity algorithm for lossy interconnect simulation," in Proc. ICCAD, Santa Clara, CA, 1991, pp. 62-65.

[22] S. Lin and E. S. Kuh, "Transient simulation of lossy interconnects based on the recursive convolution formulation," IEEE Trans. Circuits Syst. I, Fundam. Theory Appl., vol. 39, no. 11, pp. 879-892, Nov. 1992.

[23] M. J. Gander and A. E. Ruehli, "Solution of large transmission line type circuits using a new optimized waveform relaxation partitioning," in Proc. IEEE Int. Symp. Electromagnetic Compatibility, Istanbul, Turkey, 2003, pp. 636-641.

[24] T. K. Tang, M. S. Nakhla, and R. Griffith, "Analysis of lossy multiconductor transmission lines using the asymptotic waveform evaluation technique," IEEE Trans. Microw. Theory Tech., vol. 39, no. 12, pp. 21072116, Dec. 1991

[25] A. Odabasioglu, M. Celik, and L. Pileggi, "PRIMA: Passive reducedorder interconnect macromodeling algorithm," IEEE Trans. Comput.Aided Des. Integr. Circuits Syst., vol. 17, no. 8, pp. 645-654, Aug. 1998.

[26] A. Kahng and S. Muddu, "An analytical delay model for RLC interconnects," in Proc. IEEE Int. Symp. Circuits and Systems, Atlanta, GA, 1996, pp. $237-240$.

[27] Y. Gao and D. F. Wong, "Shaping a VLSI wire to minimize delay using transmission line mode," in Proc. ICCAD, San Jose, CA, 1998, pp. 611-616.

[28] J. A. Davis and J. D. Meindl, "Compact distributed RLC interconnect models. I. Single line transient, time delay, and overshoot expressions," IEEE Trans. Electron Devices, vol. 47, no. 11, pp. 2068-2077, Nov. 2000.

[29] R. E. Collin, Field Theory of Guided Waves. New York: Wiley-IEEE Press, 1990.

[30] K. D. Granzow, Digital Transmission Lines. New York: Oxford Univ. Press, 1998.

[31] M. Kamon, M. Tsuk, and J. White, "Fasthenry: A multipole-accelerated 3D inductance extraction program," IEEE Trans. Microw. Theory Tech., vol. 42, no. 9, pp. 1750-1758, Sep. 1994.

[32] W. T. Weeks, "Multiconductor transmission-line theory in the TEM approximation," IBM J. Res. Develop., vol. 16, no. 6, pp. 605-611, 1972.

[33] L. Dworsky, Modern Transmission Line Theory and Applications. New York: Wiley, 1979.

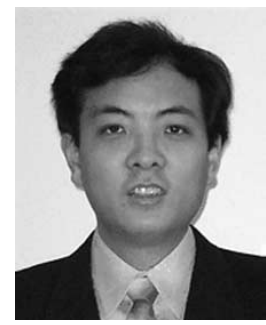

Jun Chen (S'05) received the B.S. degree in physics from Peking University, Beijing, China, in 1997 the M.S. degree in physics from Boston University, Boston, MA, in 1999, and the M.S. degree in computer engineering from the University of Wisconsin, Madison, in 2003. He is currently working toward the Ph.D. degree at the Electrical Engineering Department, University of California at Los Angeles (UCLA).

His research interests include modeling, simulation, analysis, and optimization of high-speed interconnect and electrical packaging, signal integrity, and power integrity. 


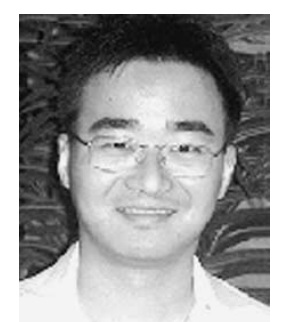

Lei He (M'99) received the B.S. degree in electrical engineering from Fudan University, Shanghai, China, in 1990 and the Ph.D. degree in computer science from the University of California at Los Angeles (UCLA) in 1999.

From 1999 to 2001, he was a Faculty Member at the University of Wisconsin, Madison. He held industrial positions with Cadence, Hewlett-Packard, Intel and Synopsys. He is currently an Assistant Professor at the Electrical Engineering Department, UCLA. His research interests include computeraided design of very large scale integration (VLSI) circuits and systems, interconnect modeling and design, programmable logic and interconnect, and power-efficient circuits and systems.

Dr. He received the Dimitris N. Chorafas Foundation Prize for Engineering and Technology in 1997, the Distinguished Ph.D. Award from the UCLA Henry Samueli School of Engineering and Applied Science in 2000, the National Science Foundation (NSF) CAREER award in 2000, the UCLA Chancellor's Faculty Development Award in 2003, and the IBM Faculty Award in 2003. 